\title{
Osteomielite: análise epidemiológica da doença no Brasil entre 2009 a 2019
}

\author{
Júlia do Carmo Santos ${ }^{1}$ (D), Ana Luísa Coelho Castro de Agüero e Ferreira ${ }^{1}$ (D), Beatriz Garcia de Paiva ${ }^{1}$ (D), \\ Heloany Verônica Quirino ${ }^{1}$, Heloísa Rodrigues Soares da Silva ${ }^{1}$, Kalyne Naves Guimarães Borges ${ }^{10}$, Jéssica \\ Silva do Carmo ${ }^{10}$, Marhara Braga de Azeredo Bastos ${ }^{1}$, Emmyli Nunes de Freitas ${ }^{2}$, Bibiana Arantes Moraes ${ }^{3}$ \\ (1).
}

\section{RESUMO}

Introdução: A osteomielite é uma infecção óssea caracterizada pela destruição progressiva do osso cortical e cavidade medular e é uma doença relevante em decorrência das suas prováveis consequências deletérias para os pacientes. Objetivo: Analisar o perfil epidemiológico de pacientes acometidos por osteomielite no Brasil durante o período de 2009 a 2019. Método: Estudo descritivo transversal, com abordagem documental, através de dados secundários coletados no Departamento de Informática do SUS (DATASUS). Os dados são referentes à morbidade hospitalar por osteomielite durante os anos de 2009 a 2019, sendo o período avaliado de janeiro de 2009 até dezembro de 2019. Resultados: Houve 183.975 internações por osteomielite no Brasil. A região Sudeste teve a maior prevalência do número de casos $(38,88 \%)$ e atingiu uma taxa de mortalidade maior que as demais regiões. Notou-se que as idades mais atingidas pela doença são entre 30 e 39 anos. Por fim, verificou-se que em todas as regiões o sexo masculino é mais acometido que o sexo feminino. Conclusão: No estudo verificaram-se alguns grupos de risco; entre eles o sexo masculino, idade entre 30-39 anos e a região Sudeste pelo elevado índice de mortalidade pela doença. A alta prevalência da doença e sua morbimortalidade elevada reforçam a necessidade de diagnóstico precoce e tratamento adequado.

Palavras-chave: Epidemiologia, Infecção, Osteomielite.

1. Universidade de Rio Verde (UniRV), Aparecida de Goiânia-GO, Brasil.

2. Universidade Vila Velha - Vila Velha, ES, Brasil.

3. Universidade Federal de Goiás (UFG), Goiânia-GO, Brasil. 


\section{INTRODUÇÃO}

A osteomielite é uma infecção óssea caracterizada pela destruição progressiva do osso cortical e cavidade medular, tendo como principal etiologia as fraturas expostas ou grandes procedimentos de reconstrução ortopédica ${ }^{1,2,3}$. Mesmo com o decréscimo da mortalidade para aproximadamente $2 \%$ atualmente, continua sendo uma doença relevante em decorrência das suas prováveis consequências, entre elas a necrose óssea ${ }^{1,3-6}$. As osteomielites têm sido classificadas de várias formas, levando-se em consideração alguns critérios como localização do processo, extensão do acometimento ósseo, estado imunológico do hospedeiro, comorbidades e tipo de agente etiológico causador $^{7-11}$. As bactérias aderem ao osso devido às adesinas, são elas que vão contribuir para a gravidade ${ }^{5,6,7,8}$.

O diagnóstico pode ser realizado pela história e exame clínico, a despeito da sofisticação atual dos métodos de imagem. Em relação à sintomatologia pode ocorrer febre, calafrios, abcessos, edema e eritema local ${ }^{1,3,9}$. Além da clínica, é importante investigar história de traumas, procedimentos cirúrgicos, fraturas e infecções, e ao exame físico é importante procurar por diminuição dos pulsos, úlceras perfurantes, celulites e fístulas. ${ }^{10,11,12}$

Para auxiliar no diagnóstico solicita-se alguns exames, laboratorialmente faz-se o isolamento do agente, hemocultura, proteína $\mathrm{C}$ reativa, hemograma, VHS, dosagem da alfa-glicoproteína ácida. Exames de imagem que podem colaborar para a investigação são radiografia óssea, que apresenta alterações após $010^{\circ}$ dia do início da doença, Tomografia Computadorizada, Ressonância Magnética e Ultrassonografia da lesão. Após o diagnóstico, o manejo da doença engloba medidas de higienização, cuidado da ferida, antibioticoterapia a depender da suspeita do germe e manejo cirúrgico $5,13,14,15$.

O quadro da doença em sua fase inicial é inespecífico, mas se bem avaliado, correlacionando a história clínica com o exame físico e a epidemiologia da osteomielite pode-se realizar o diagnóstico precocemente e assim evitar a disseminação da doença e a piora do quadro do paciente. ${ }^{16,17} \mathrm{O}$ tratamento tem eficácia e tempo de início diretamente relacionadas, sendo que quando mais precoce é iniciado, maior a efetividade e resolutividade. Levando em consideração a morbimortalidade da doença, suas complicações e o início do tratamento, faz-se de suma importância o estudo epidemiológico da enfermidade, visto que é uma doença que pode trazer graves sequelas, mas que se diagnosticada precocemente pode evoluir com prognóstico altamente favorável. ${ }^{12,13,14}$

As osteomielites podem ser causadas por diversos agentes etiológicos, sendo os mesmos diretamente relacionados ao prognóstico da morbidade e ao seu tratamento com antibioticoterapia, interferindo na escolha da medicação, necessidade ou não de associação e o tempo de tratamento. ${ }^{13,14}$ Podemos citar os seguintes agentes etiológicos: Staphylococcus aureus, Staphylococcus aureus multirresistentes, Enterobacter sp., Klebsiella sp., Acinetobacter sp., Pseudomonas $s p$. e as com acometimento polimicrobiano. ${ }^{13,14,15,18}$

Tendo em vista a importância econômica e social da doença é importante realizar pesquisas, como o presente estudo para identificar a população mais susceptível a morbidade e assim intervir de forma efetiva e resolutiva. O objetivo deste estudo é analisar o perfil epidemiológico de pacientes acometidos por osteomielite no Brasil durante o período de 2009 a 2019, para definir a epidemiologia dos grupos de risco da morbidade, possibilitando um melhor conhecimento acerca da doença e posteriormente permitindo intervenções de forma a evitar prejuízos tanto para o paciente, quanto para o sistema de saúde.

\section{MÉTODOS}

Foi realizado um estudo descritivo transversal, com abordagem documental, através de dados secundários coletados no Departamento de Informática do Sistema Único de Saúde (DATASUS) ${ }^{19}$. O DATASUS é um departamento que tem como missão promover a modernização através da tecnologia da informação para apoiar o Sistema Único de Saúde (SUS) e que disponibiliza informações da Assistência à Saúde da população, da Rede Assistencial, das redes hospitalares e ambulatoriais, do cadastro de saúde, dos recursos financeiros e de dados demográficos e socioeconômicos.

Os dados coletados para o presente estudo são referentes à morbidade hospitalar por osteomielite durante os anos de 2009 a 2019. Para a realização da atual pesquisa foram inseridos dados secundários disponibilizados no DATASUS, através da pesquisa 
pelo código da Classificação Internacional de Doenças e Problemas Relacionados à Saúde (CID-10), sendo utilizado o código M86, referente à Osteomielite.

A pesquisa pelo CID-10 revelou dados referentes à morbidade que foram disponibilizados na plataforma e para realização da pesquisa foram selecionados os dados com base em critérios de inclusão e exclusão, sendo os mesmos citados a seguir. Foram critérios de inclusão os dados secundários da morbidade referentes ao período de janeiro de 2009 a dezembro de 2019; dados do perfil de acometimento pela doença, englobando sexo, faixa etária e acometimento por região de internação; quantidade de internações e quantidade de óbitos pela doença. Foram critérios de exclusão os dados disponibilizados que não foram coletados devido a internações pelo CID-10 M86.

Os dados obtidos na pesquisa forma selecionados obedecendo aos critérios citados no estudo e foram esquematizados em tabelas de forma a permitir comparação das internações e óbitos de forma anual, por gênero, faixa etária e também região. Após a esquematização em tabelas, tornouse possível a análise quantitativa e descritiva dos dados, definindo o perfil epidemiológico da população brasileira quando se aborda a osteomielite.

\section{RESULTADOS}

Durante o período estudado, houve 183.975 internações por osteomielite no Brasil. A região Sudeste teve a maior prevalência do número de casos $(38,88 \%)$, seguido pelas regiões Nordeste $(30,64 \%)$, Sul $(15,01 \%)$, Centro-Oeste $(8,42 \%)$ e Norte $(7,03 \%)$. Notou-se que o Sudeste atingiu uma taxa de mortalidade de 1,6 óbitos para cada 100 pacientes internados, seguido de taxas de 1,16 no Nordeste, 0,99 no Sul, 0,81 no Centro-Oeste e 0,37 na região Norte.

A análise nacional da mortalidade da osteomielite obteve uma taxa de mortalidade da doença de 1,26 óbitos para cada 100 pacientes internados. Já em relação à faixa etária, notou-se que as idades mais atingidas pela doença foram entre 30 e 39 anos ( $16,25 \%$ dos casos) e considerando que o intervalo de idade de 20 a 49 anos abrange quase a metade, $47,54 \%$, dos casos no país.
Verificou-se que em todas as regiões analisadas e estudadas o sexo masculino foi mais acometido que o sexo feminino. O gênero masculino é responsável por $70,87 \%$ dos casos de internação no Brasil por osteomielite.

\section{DISCUSSÃO}

Durante os anos de 2009 a 2019 houve 183.975 internações por osteomielite no Brasil. No presente estudo verificaram-se alguns grupos de risco; entre eles o sexo masculino, idade entre 3039 anos e a região Sudeste pelo elevado índice de mortalidade pela doença. Por esse fato, estudos e pesquisas que envolvam a prevalência, mortalidade e necessidade de hospitalização da osteomielite se faz tão importante e fundamental.

Enfatizando a necessidade de atualização dos dados secundários de forma contínua, a Secretaria de Saúde por meio da Vigilância Epidemiológica deve possibilitar o acompanhamento dos dados epidemiológicos e assim interferir de maneiras efetivas incentivando as unidades de assistência à saúde a seguirem o fluxo de atendimento preconizado para a doença, objetivando evitar o aumento da incidência de casos e um melhor prognóstico para os pacientes acometidos. , $3,4,5^{-1}$

Quando analisados os dados na presente pesquisa, chegamos a conclusões epidemiológicas de maior acometimento por delimitação regional, sendo a região Sudeste a que teve maior prevalência. Tal fato pode se relacionar à densidade populacional de cada região, onde proporcionalmente os estados com o maior número de habitantes obtém maior chance de ter maior prevalência da doença. ${ }^{1,3,15} \mathrm{~A}$ região Sudeste teve a maior prevalência do número de casos de osteomielite, seguido pelas regiões Nordeste, Sul, Centro-Oeste e Norte, e quando se fala em dados do Instituto Brasileiro de Geografia e Estatística (IBGE) ${ }^{16,17}$ a região com maior densidade populacional é a Sudeste, seguida da Sul, Nordeste, Centro-Oeste e Norte. ${ }^{17,18}$

Abordando as regiões do país, nota-se ainda que o Sudeste atingiu uma taxa de mortalidade maior quando comparada as outras regiões, seguida da região Nordeste, Sul, Centro-Oeste e Norte. Podese atribuir esses resultados como provável causa da discrepância na prática de medidas de higiene, 
a prevalência de procedimentos cirúrgicos, doenças vasculares, traumas, uso de próteses ortopédicas e infecções, sendo essas as principais causas de osteomielite na faixa etária mais acometida que necessita de internação.

Em todas as regiões analisadas pelo estudo o sexo masculino é mais acometido que o sexo feminino. Essa prevalência é evidenciada em artigos de epidemiologia que relacionam a osteomielite a eventos traumáticos, como acidentes e procedimentos cirúrgicos, evidenciando a conformidade dos resultados do estudo com as demais literaturas. ${ }^{18,20,21,22}$

O presente estudo encontrou uma percentagem de $70,87 \%$ de acometimento no sexo masculino, em contrapartida ao estudo do tipo transversal, com coleta de dados mediante questionário, a partir de uma revisão de prontuário, que evidenciou $78,95 \%$ dos casos em homens. ${ }^{15,20,22,23}$ Mantendose essa proporção de mais de $70 \%$ nos estudos avaliados. ${ }^{15,18,20,21,22}$

O sexo masculino obteve maior número de notificações em todo o Brasil e durante todos os anos das pesquisas utilizadas para realização do artigo, porém, considerando os pacientes acima de 80 anos, há inversão do perfil de internações por osteomielite, apresenta predominância no sexo feminino, levando em consideração que nesta faixa etária o número de mulheres aumenta sobre o de homens, existe a relação direta entre o aumento da idade e um crescimento do número de comorbidades para este grupo. ${ }^{15,18,20-23} \mathrm{Com}$ relação à pesquisa, a faixa etária mais atingidas pela doença foi entre 30 e 39 anos, considerando que o intervalo de idade de 20 a 49 anos abrange quase a metade dos casos no país. Estudos evidenciaram que a faixa etária mais acometida está entre os 30 aos 59 anos, sendo a parcela economicamente ativa. ${ }^{15,22}$

Em relação ao perfil epidemiológico da osteomielite e a sua relação com as fraturas, nota-se associação de fatores como o grau de contaminação da fratura, as condições nutricionais do indivíduo, o tempo desprendido entre o traumatismo e o correto atendimento. ${ }^{15,24} \mathrm{~A}$ média de idade dos pacientes que apresentaram osteomielite relacionada a fraturas apresenta uma média de 35 anos, sendo a maioria do sexo masculino. ${ }^{15,20,21,24,25}$ Os prováveis motivos para a ocorrência de osteomielite em fraturas expostas podem estar vinculados ao desperdício de tempo do momento do acidente até o ato operatório inicial, já comentado, características próprias dos ferimentos, elevado grau de contaminação, acidente em zona rural, primeiro atendimento, e condições de remoção e transporte. ${ }^{15,20-25}$

Na realização do estudo é possível identificar uma limitação que pode interferir nos dados e em sua avaliação integral, visto que os dados secundários dependem de um fluxo para serem registrados de maneira adequada e, assim, poderem ser efetivamente analisados. $O$ estudo tem barreiras de informação devido à possibilidade de falhas dos registros dos dados e isso deve ser levado em consideração. No entanto, foram avaliados vários anos na pesquisa o que possibilita um resultado mais fidedigno a realidade.

\section{CONCLUSÃO}

O estudo objetivou analisar o perfil epidemiológico da população mais susceptível a osteomielite e, assim, intervir antes da incidência da doença e suas complicações. O perfil epidemiológico brasileiro de acometimento pela osteomielite é composto pelo sexo masculino, com idade entre 30-39 anos e com maior índice de mortalidade pela doença na região Sudeste.

Identificando os fatores de risco e o perfil epidemiológico da morbidade, torna-se relevante a implementação de políticas públicas de saúde voltadas ao cuidado de pacientes do sexo masculino, adultos jovens e que tenham tido algum trauma com presença de lesões que possam evoluir para osteomielite.

A doença ainda é muito frequente, mesmo com as medidas tomadas para evitar que os traumas e infecções evoluam para piores prognósticos. A dificuldade do tratamento da osteomielite é evidenciada na múltipla gama de antibioticoterapias empregadas nesses casos e ampla resistência que vem sendo adquirida. São muitas as dificuldades que envolvem essa comorbidade, e a alta prevalência da doença e sua morbimortalidade elevada reforçam a necessidade de diagnóstico precoce e tratamento adequado. 


\section{REFERÊNCIAS}

1. Lima, A.L.M., Oliveira, P. R. D. Osteomielites. Faculdade de Medicina da Universidade de São Paulo - São Paulo, 2003.

2. Lima, A. L. M. Infecções em Próteses Articulares. Faculdade de Medicina da Universidade de São Paulo São Paulo, 2004.

3. Grinbaum, R. S. Tratamento de Infecção Hospitalar Uma Abordagem Prática.- São paulo; Planmark, 2005, Pág: 50- 51

4. Cabanella, M.E., Sim, F.H., Beabout, J.W. et al. Osteomyelitis appearing as neoplasm. Arch Surg 109: 68-72, 1974.

5. Carazzato, J.G., Camanho, G.L., Faga, A. et al. Tratamento da osteomielite hematogênica aguda. Rev Bras Ortop 13: 121-128, 1978.

6. Ezra, E., Khermosh, O., Assia, A. et al. Primary subacute osteomyelitis of the axial and appendicular skeleton. J Pediatr Orthop 1: 148-152, 1993.

7. Green, M.E., Beauchamp, R.D. \& Griffin, P.D.: Primary subacute osteomyelitis. J Bone Joint Surg [Am] 63: 107-114, 1981.

8. Harris, N.H. \& Kirkaldy-Willis, W.H.: Primary subacute pyogenic osteomyelitis. J Bone Joint Surg [Br] 47: 526532, 1965.

9. Jones, N.S., Anderson, D.J. \& Stiles, P.J.: Osteomyelitis in a general hospital. J Bone Joint Surg [Br] 69: 779783, 1987.

10. King, D.M. \& Mayo, K.M.: Subacute hematogenous osteomyelitis. J Bone Joint Surg [Br] 51: 458-463, 1969.

11. Lindenbaum, S. \& Alexander, H.: Infections simulating bone tumors. Clin Orthop 184: 193-203, 1984.

12. Stephens, M.M. \& MacAuley, P.: Brodie's abscess. Clin Orthop 234: 211-216, 1988.

13. Hamdy, R.C., Lawton, L., Carey, T. et al: Subacute hematogenous osteomyelitis: are biopsy and surgery always indicated? J Pediatr Orthop 16: 220-223, 1996.

14. Hungria Filho, J.S.: Os antibióticos no tratamento da osteomielite. Rev Bras Ortop 7: 25-28, 1982.

15. Villa PEA, Nunes TR, Gonçalves FP, Martins JS, Lemos GSP, Moraes FB. Avaliação clínica de pacientes com osteomielite crônica após fraturas expostas tratados no Hospital de Urgências de Goiânia, Goiás. Rev Bras Ortop. 2013; 48(1): 22-28.

16. Instituto Brasileiro Geografia Estatística - IBGE. Censos Demográficos. Disponível em: http://www.ibge.gov. br/home/estatistica/populacao/defaultcenso2000. shtm [Acessado em 26 de julho de 2020].
17. ATLAS nacional do Brasil Milton Santos. Rio de Janeiro: IBGE, 2010. 307 p.

18. Neto, F.C.J.; Ortega, CS. Goiani E.O. Estudo epidemiológico das infecções osteoarticulares em crianças. Acta Ortop Bras. 2018;26(3):201-5.

19. Brasil, Ministério da Saúde (MS). Banco de dados do Sistema Único de Saúde - Departamento de Informática do SUS (DATASUS), Informações de Saúde, Rede assistencial. Disponível em: http://www2.datasus.gov. br/DATASUS/index.php?area $=0203 \& i d=6927 \& V O b j=h$ ttp://tabnet.datasus.gov.br/cgi/deftohtm.exe?sih/cnv/ nr [Acessado em: 26 de julho de 2020].

20. Müller SS, Sadenberg T, Pereira GJC, Sadatsune T, Kimura EE, Novelli Filho JLV. Estudo epidemiológico, clínico e microbiológico prospectivo de pacientes portadores de fraturas expostas atendidos em hospital universitário. Acta Ortop Bras. 2003;11(3):158-69.

21. Arruda LRP, Silva MAC, Malerba FG, Fernandes MC, Turíbio FM, Matsumoto MH. Fraturas expostas: estudo epidemiológico e prospectivo. Acta Ortop Bras. 2009;17(6):326-30.

22. Moore TJ, Mauney C, Barron J. The use of quantitative bacterial counts in opens fractures. Clin Orthop. 1989; (248):227-30.

23. Souza CS, Bandeira LLB, Aguiar MC, Cruz CF, Aragão IPB, Neto JDS. Análise do perfil de atendimento por osteomielite em pacientes acima de 60 anos. Rev Soc Bras Clin Med. 2019;17(2):71-5.

24. Kinik H, Karaduman M. Cierny-Mader Type III chronic osteomyelitis: the results of patients treated with debridement, irrigation, vancomycin beads and systemic antibiotics. Int Orthop. 2008;32(4):551-8.

25. Müller SS, Sadenberg T, Pereira GJC, Sadatsune T, Kimura EE, Novelli Filho JLV. Estudo epidemiológico, clínico e microbiológico prospectivo de pacientes portadores de fraturas expostas atendidos em hospital universitário. Acta Ortop Bras. 2003;11(3):158-69. 
Osteomielite: análise epidemiológica da doença no Brasil 
Autor Correspondente:

Júlia do Carmo Santos

juliadocarmosantos@hotmail.com

Editor:

Prof. Dr. Marcelo Riberto

Recebido: 17/09/2020

Aprovado: 09/02/2021 